Itinéraires Itinéraires

Littérature, textes, cultures

2012-3 | 2013

Lire les villes marocaines

\title{
Représentation littéraire de Fès
}

\section{Anouar Ouyachchi}

\section{OpenEdition}

\section{Journals}

Édition électronique

URL : http://journals.openedition.org/itineraires/984

DOI : 10.4000/itineraires.984

ISSN : 2427-920X

Éditeur

Pléiade

Édition imprimée

Date de publication : 1 juillet 2013

Pagination : 111-126

ISBN : 978-2-343-01183-7

ISSN : $2100-1340$

Référence électronique

Anouar Ouyachchi, «Représentation littéraire de Fès », Itinéraires [En ligne], 2012-3 | 2013, mis en ligne le 01 décembre 2012, consulté le 02 mai 2019. URL : http://journals.openedition.org/itineraires/984 ; DOI : 10.4000/itineraires.984

\section{(c) (1) $\Theta$}

Itinéraires est mis à disposition selon les termes de la licence Creative Commons Attribution - Pas d'Utilisation Commerciale - Pas de Modification 4.0 International. 


\title{
Représentation littéraire de Fès
}

\begin{abstract}
Literary works give a specific account of the collective discourses continuously held on a town like Fes. They contribute to producing a steady and permanent image of the town, and they reinforce its Big Story. Nevertheless, when one examines more closely how the literary representation operates inside these works, one notices that the Big Story never stops splitting up and reconstructing itself again according to history, gender, sex and the way in which people looked at this town... How can this articulation of the personal to the collective enable a representation of Fes?
\end{abstract}

Keywords : Fes, representation, Big Story, literary genre

Mots clés : Fès, représentation, Grand Récit, genre littéraire

Pour essayer de mieux comprendre le rapport ville/littérature, il faut se rappeler deux choses : premièrement, dans un récit, l'espace n'est pas seulement ce qui permet d'ancrer, par un « effet de réel », intrigue et personnages dans des lieux facilement identifiables en fournissant des repères au lecteur; il est aussi le produit d'une représentation littéraire dont le degré symbolique varie d'une œuvre à l'autre. Même dans le cas du récit le plus mimétique qui soit, où l'auteur sacrifie au principe de vérisimilitude, l'agencement littéraire des réalèmes n'exclut pas un travail de modélisation et de reconfiguration. Deuxièmement, les œuvres littéraires, lorsqu'elles investissent des villes du monde réel, ne nous en livrent qu'une image très réduite. D'abord, parce que la littérature n'a pas d'autre possibilité : malgré tous les rapprochements qu'elle peut établir entre ville littéraire et référentiel urbain, celle-ci « ne peut sérieusement modifier la contrainte qui est la sienne d'une représentation métonymique et successive des dénotations spatiales ${ }^{1} »$; l'espace urbain littéraire est un

1. Jean-Christophe Valtat, «Modèles physiques de l'espace littéraire : champ relativiste, champ quantique », dans J. Vion-Dury et al. (dir.), Littérature et espaces, Limoges, Presses universitaires de Limoges, 2003, p. 91. 
espace « construit formellement sur la valorisation de lieux privilégiés ${ }^{2}$ ». Ensuite, il n'y a pas de ville réelle en dehors des représentations cognitives qui en favorisent l'appropriation et la compréhension de façon schématique et simplifiée. Autrement dit, « l'espace urbain, tel que l'individu l'apprend, le perçoit et le connaît, est déjà un espace (re)présenté à lui par les pratiques urbaines, c'est-à-dire qu'il est déjà un espace de représentation socialement, idéologiquement posé ${ }^{3} »$. Ainsi, les œuvres littéraires n'ont pas affaire à un continuum urbain indifférencié qu'elles découpent et structurent à leur manière. Outre les strates intertextuelles, des représentations cognitives de la ville viennent s'interposer comme des espaces de médiation entre le référentiel urbain et les villes symboliques de la littérature en filtrant toute l'activité de mise en langage.

Étudier la représentation littéraire d'une ville marocaine comme Fès, en tenant compte de ces deux remarques liminaires, serait ouvrir un immense chantier où les multiples récits de la ville opèrent une projection de l'espace urbain infiniment variable au gré des périodes historiques, de la nature du regard (exogène ou endogène), des genres littéraires, du sexe, de la classe sociale... En examinant des textes d'auteurs marocains ou occidentaux, nous tenterons aussi bien d'étudier quelques-unes des modalités du fonctionnement de la représentation littéraire de Fès que de cerner le Grand Récit de cette ville, construit et déconstruit au gré des différentes expériences littéraires.

\section{Fès ou la ville des expressions du moi}

Peut-on parler de la représentation littéraire de Fès sans pour autant prendre en considération la spécificité des genres dans lesquels elle s'inscrit? La relation entre l'espace et le système générique se révèle beaucoup plus étroite qu' on ne le pense. Non seulement « chaque genre littéraire a sa géographie, sa géométrie presque ${ }^{4} \gg$ qui lui permet de façonner les lieux et de les articuler selon des lois et des codes particuliers, mais le style et le contenu des œuvres dépendent aussi, pour une grande part, du cadre spatial élu par l'écriture; on peut penser que « chaque espace détermine, ou tout au moins, encourage, un type d'histoire différent ${ }^{5}$ ». Ainsi, l'une des spécificités de Fès, à l'instar d'autres villes marocaines, est sa propension à générer une littérature de l'intime où la relation entre le moi et l'espace constitue la pierre angulaire de la représentation littéraire. On ne peut nier qu'il existe des fictions de Fès, néanmoins la ville se trouve surtout associée à la littérature de voyage et à la forme autobiographique.

2. Ibid., p. 91 (c'est l'auteur qui souligne).

3. Martial Fauteux, «Représentation de la ville», Cahiers de géographie du Québec, vol. $21, \mathrm{n}^{\circ} 52,1977$, p. $83-103$, p. 85.

4. Franco Moretti, Atlas du roman européen (1800-1900), Paris, Seuil, 2000, p. 208.

5. Ibid., p. 83. 
Depuis la fin du XIX ${ }^{e}$ siècle, époque marquée en Occident colonial par une étroite imbrication entre science géographique, littérature de voyage et conquête militaire, diplomates, journalistes, reporters et écrivains voyageurs français ne cessent de cartographier la cité impériale en livrant des représentations à la croisée du savoir et de l'imaginaire. Pierre Loti, Eugène Aubin, André Chevrillon, les frères Tharaud, pour ne citer que ceux-ci, ont tous été inspirés par la ville de Fès. Au-delà du fait que, dans le cas des récits de ces écrivains, «le moi projette dans l'espace sa propre mythologie de l'histoire de la nation à laquelle il appartient ${ }^{6} »$, la description demeure, du point de vue littéraire, l'outil privilégié permettant à l'écrivain voyageur de construire le référentiel urbain et de le structurer. Une fièvre de tout saisir s'empare du voyageur dont les sens sont sans cesse interpellés. Toute la ville, dont les différents lieux sont découverts progressivement, de façon affichée ou clandestinement (Loti déguisé en Maure), est littéralement verbalisée selon une rhétorique orientaliste où l'exotique et/ou le scientifique opèrent comme des moyens $d^{\prime}$ " "annexion" littéraire ${ }^{7} » d$ 'une contrée lointaine ou d' " occupation d'espaces "vides" " ", tant à explorer qu'à « inventer ${ }^{9} »$. D'ailleurs, il suffit de jeter un rapide regard sur les livres ou les anthologies de récits de voyages consacrés totalement ou partiellement à Fès ${ }^{10}$ pour avoir une idée du fonctionnement de cette rhétorique. Grâce à une poétique des sens où le regard est sans cesse privilégié, le voyageur multiplie les descriptions panoramiques et détaillées de la ville avec variation d'échelle et de position. Ainsi, aux vues d'ensemble que le voyageur peut avoir de Fès, soit de loin au moment de son approche, soit à partir d'une terrasse ou d'un point élevé situé aux abords de la ville, et qui nous sont livrées à travers des descriptions où le paysage urbain se fond souvent dans le paysage naturel, s'ajoutent des tableaux pittoresques de lieux et de scènes consacrés comme topoï presque obligés du genre : la médina et ses souks, la mosquée Qarawiyine, le sanctuaire Moulay Idriss, le mellah, les palais... les descriptions faisant souvent ressortir l'image d'une vieille cité médiévale insensible au progrès. Cette appropriation de la ville et sa conquête littéraire, dans une sorte de va-et-vient entre le général et le particulier, l'ensemble et le détail, se modulent et se nuancent selon les états d'âme d'un moi dont les sentiments et les émotions ne cessent de varier. Chez les frères Tharaud, par exemple, après un premier regard sur la ville,

6. Alet Valero, « Le moi et l'espace dans les récits de voyage », dans J. Soubeyroux (dir.), Le Moi et l'Espace : autobiographie et autofiction dans les littératures d'Espagne et d'Amérique Latine, Saint-Étienne, Publications de l'Université de Saint-Étienne, 2003, p. 84.

7. János Riesz, «Astres et désastres ». Histoire et récits de vie africains de la colonie à la postcolonie, Hildesheim, Georg Olms, 2009, p. 103.

8. Ibid.

9. Ibid.

10. En guise d'exemple, citons Alain Lavaud, Fès, années 20 [2000], Casablanca, La Croisée des chemins, 2007 et Natacha Potier, Dix-sept regards sur le Maroc, Casablanca, Eddif, 2006. 
cherchant à restituer l'émotion et la " naïveté » du premier contact, prend place une nouvelle description où s'exprime la nostalgie des premières impressions :

Je suis revenu par la suite si souvent à cet endroit d'où j'ai vu Fès pour la première fois, qu'il m'est à peu près impossible de restituer dans son intégrité l'impression du moment. Elle est là, en moi, je le sais : en la cherchant, les yeux fermés, je crois que je vais la saisir, mais aussitôt d'autres images viennent se jeter à la traverse. La longue descente immaculée ne m'apparaît plus toute blanche. Je vois les taches, les moisissures de cette ville humide. La vie des ruelles invisibles, qui craquellent en tous sens cette masse serrée, me semble remonter à la surface et se dessiner sur ces blancheurs. Partout je mets des noms, des visages. Ma première impression est devenue pareille à un manuscrit surchargé, qui peut-être ne vaut pas la page primitive ${ }^{11}$.

Chez Pierre Loti, on peut déceler toute une palette d'états d'âmes contradictoires et instables allant de la déception du premier moment au sentiment de paix que procure, par exemple, la vision de la ville se métamorphosant brusquement, par un beau jour de soleil, sous le regard de l'écrivain voyageur la redécouvrant du haut de sa terrasse, en une cité radieuse qui n'a plus rien de sombre et de « fanatique ». Cependant, outre l'image d'une ville « infiniment vieille » et mortuaire, prise dans le « suaire de l'Islam », pour Loti, Fès est généralement un lieu gris et sombre inspirant la tristesse. D'ailleurs, de toutes les visions que la ville avait inspirées à l'écrivain, c'est bien celle d'un Fès sinistre qui refera surface avec force au moment du départ pour Meknès :

Fez s'éloigne sur ces mêmes fonds sombres, prend ces mêmes aspects sinistres qui nous étaient restés dans la mémoire depuis sa première apparition au matin de notre arrivée. En nous retournant, longtemps nous pouvons voir encore, au pied de ses murailles presque noires, les rangées de petits cônes blancs comme neige qui sont le camp du très saint calife... Des teintes tristes partout; les passants enveloppés de laine, les chameaux, les ânons, tout ce qui fait le va-et-vient entre les deux villes par ce même et unique sentier a des couleurs terreuses, brunâtres et grises ${ }^{12}$.

Pour les écrivains marocains, l'autobiographie demeure la forme littéraire de l'appropriation de la ville par excellence. On peut même dire que l'une des spécificités de la littérature marocaine francophone, depuis Le Passé simple de Driss Chraïbi, est sa tendance particulière à associer le moi et l'espace en investissant la ville comme un lieu d'expression et de négociation identitaires. Il suffit de jeter un coup d'œil sur certains récits fondateurs des années 1960 et 1970 pour se rendre compte combien la réflexion sur les questions du moi et de l'hybridité culturelle étaient intimement liées à

11. Jérôme et Jean Tharaud, Fès ou les bourgeois de l'Islam [1930], Rabat, Marsam, 2008, p. 17.

12. Pierre Loti, Au Maroc [1890], Casablanca, Eddif, 2008, p. 281. 
une projection de la ville comme un lieu identitaire que le sujet postcolonial marocain cherche à se réapproprier à la lumière des discontinuités de l'histoire. Soucieux de rompre avec les clichés des écrivains orientalistes, qui n'avaient vu dans le Maroc, et ceci à des degrés divers, qu'un objet de fantasmes et de mythifications, les auteurs de cette période avaient opéré, dans le sillage de l'expérience de décolonisation culturelle menée par la revue Souffles (1966-1972), un déplacement dans la représentation littéraire de la ville marocaine. Du point de vue littéraire, ce déplacement d'un genre à l'autre, accompagné d'un glissement du regard exogène au regard endogène, a plusieurs implications dont la plus importante est l'investissement de la ville, vécue de l'intérieur, comme le miroir d'une société en pleine mutation. Cependant, en lisant les récits à caractère autobiographique des auteurs marocains, le lecteur ne manquera pas de sentir un émoussement des sens et un rétrécissement de l'espace urbain qui devient beaucoup moins étendu que dans le récit de voyage. En outre, contrairement aux textes des voyageurs où le regard opère fréquemment des va-et-vient entre le proche et le lointain, les récits autobiographiques ont plutôt tendance à réduire les repères visuels qui permettent d'ancrer les microlieux dans des paysages très vastes. Dans $L e$ Fond de la jarre ${ }^{13}$ d'Abdellatif Laâbi, par exemple, le Fès que nous découvrons, au gré des déambulations du jeune narrateur, surnommé Namouss, est surtout celui des lieux correspondant à des zones de la ville accessibles et appropriées psychologiquement pendant l'enfance de l'auteur. Aussi ne dépasse-t-il pas souvent les frontières du quartier :

Le territoire de Namouss est un mouchoir de poche. Il se limite au quartier des Kairouanais, et encore. Quant à l'autre quartier, celui des Andalous, Namouss n'y a pour ainsi dire jamais mis les pieds. Pour lui, comme pour la plupart de ses camarades, c'est presque un pays étranger où il ne fait pas bon de s'aventurer. Là-bas vivent des bandes d'enfants hostiles qu'on ne rencontre que lors de batailles sporadiques ${ }^{14}$.

Cette réduction de l'espace urbain aux seuls lieux familiers n'implique pas pour autant une disparition de l'événementiel dans Le Fond de la jarre. Lieu historiquement défini par l'histoire du mouvement nationaliste et la lutte pour l'indépendance, le Fès que décrit Laâbi, quoique réduit à un territoire d'enfant, permet d'engendrer de nouvelles expériences. Bon gré mal gré, la petite histoire du narrateur, enfermée dans sa propre temporalité cyclique, s'y trouve mêlée à la grande histoire mouvementée de la nation. Par ailleurs, malgré ses dimensions réduites, la ville dans Le Fond de la jarre n'est jamais un espace désémantisé. Outre sa construction comme lieu d'émergence d'une conscience politique, celle-ci se donne aussi à voir comme une immense scène de théâtre offrant au regard un spectacle permanent de personnages marginaux auxquels le jeune narrateur emboîte souvent

13. Abdellatif Laâbi, Le Fond de la jarre, Paris, Gallimard, 2002.

14. Ibid., p. 94. 
le pas pour redécouvrir à chaque fois son territoire. Émergent ainsi, progressivement, dans une sorte de corps à corps avec la médina et au rythme d'une errance centripète, qui finit toujours par mener au sanctuaire de Moulay Idriss puis à la Qaraouiyine, les lieux d'un paysage urbain familier que le narrateur connaît « souk par souk, place par place, ruelle par ruelle ${ }^{15}$ ». La relation avec la ville nouvelle, associée généralement aux Nazaréens, est plutôt de l'ordre de la fascination. Lorsque le narrateur entre à l'école franco-musulmane, il découvre des livres avec des images mystérieuses qui semblent issues d'un autre monde, et c'est la ville nouvelle de Fès, dans laquelle il ne s'est jamais aventuré auparavant, qui vient incarner pour lui ce monde livresque dont le spectacle s'est brusquement substitué à celui à ciel ouvert de la médina. Il va de soi que cette relation ville/système générique se module en fonction d'autres critères tels que le sexe ou le groupe social. Dans un roman autobiographique, d'abord publié en anglais en 1994, puis traduit en français sous le titre Rêves de femmes ${ }^{16}$, l'écrivaine et essayiste marocaine Fatéma Mernissi nous livre une autre image du Fès colonial des années 1940, construite selon les codes d'une géographie culturelle des sexes. Avec ce récit, l'auteure mène une réflexion sur l'identité sexuelle, à travers une exploration de la ville, lieu traversé par des frontières et des discours normatifs qui organisent les rapports entre l'homme et la femme dans un Maroc en pleine mutation. L'univers des femmes, au sein duquel nous introduit Mernissi, ne dépasse pas le portail de la maison. « Petit dieu de seuil ${ }^{17} \gg$, ce portail a pour fonction de séparer, comme l'explique la narratrice, « le harem des femmes des étrangers de la rue ${ }^{18} »$. Dérobant souvent la ville au regard de son lecteur, puisqu'elle est rarement associée aux expériences des femmes dont il raconte la vie, ce roman semble frappé de cécité, confiné dans les murs du harem. C'est un récit du dedans et de l'espace clos, un récit de la non-ville où le dehors est vécu sur le mode de l'hypothétique et du souhait :

\footnotetext{
Je me lèverais à l'aube, disait ma mère de temps en temps, si seulement je pouvais aller me promener au petit matin quand les rues sont désertes. La lumière doit être bleue, ou rose vif peut-être, comme au coucher du soleil. Quelle est la couleur du matin dans les rues désertes et silencieuses? Personne ne répondait à ses questions ${ }^{19}$.
}

Dans ce harem-forteresse, toutes les fenêtres donnent sur la cour; les seules fleurs connues sont celles des brocarts et des rideaux; le ciel, piégé par les hommes dans l'encadrement de la cour, est décrit comme carré et terne.

\section{Ibid.}

16. Fatéma Mernissi, Rêves de femmes : contes d'enfance au harem, Casablanca, Le Fennec (pour la version française commercialisée au Maroc), 1997.

17. Ibid., p. 200.

18. Ibid., p. 31.

19. Ibid., p. 31-32. 
Après cette première vision de Fès telle qu'elle apparait à la croisée de deux genres et de deux regards (exogène et endogène), c'est-à-dire mobile et fuyante, il serait à présent intéressant de s'interroger sur la manière dont la ville permet de fixer le sens et de le rendre permanent en le préservant de tout ce qui menace de l'atomiser.

\section{Le Grand Récit de Fès}

Comme les nations, les villes sont aussi des narrations. Autrement dit, dans la trame des discours mouvants qu'on entretient sur les cités, s'isole toujours un noyau dur permanent qui fonctionne comme un Grand Récit où la perception de l'espace réel se trouve souvent façonnée par plusieurs éléments d'ordre historique, religieux, identitaire voire légendaire et mythique. Ainsi, même si les représentations d'une ville changent selon les individus et les époques, ce Grand Récit persiste et resurgit comme un repère servant aussi bien de lieu de mémoire aux membres d'une communauté imaginée que de raccourci à tous ceux qui renvoient à cette communauté et la désignent. Qu'en est-il donc du Grand Récit de Fès?

Avec Tanger et Marrakech, Fès est peut-être l'une des villes marocaines les plus surreprésentées en littérature. En outre, un grand nombre d'écrivains marocains, arabophones aussi bien que francophones, sont natifs de Fès ou lui sont associés, dans l'esprit du lecteur, d'une façon ou d'une autre. Pour ce dernier, des noms comme ceux d'Ahmed Sefrioui, Driss Chraïbi, Abdellatif Laâbi, Tahar Ben Jelloun, Fatéma Mernissi, Abdelkrim Ghallab ou Mohammed Berrada sont solidement ancrés dans cette ville. Comme Tanger, Fès possède ses écrivains, Marocains aussi bien qu'étrangers. Pourtant, le mythe de Fès n'est pas aussi prégnant que celui de Tanger. Faut-il donc plus que des mots pour transformer la pierre en mythe? Ou la mythologie moderne aura-t-elle plutôt tendance à se confondre avec un discours de la marge et de l'interstice, c'est-à-dire tout ce que la ville de Fès ne représente pas?

Il existe certainement un récit de Fès. Ce récit, tel qu'on peut le reconstituer, à la croisée des discours social et littéraire, se donne généralement à lire comme celui d'une ville du passé et de la centralité par excellence. Contrairement à Tanger, par exemple, ville interlope, dont toute la mythologie s'est constitué autour des notions de marge et de transgression, Fès est plutôt une ville du pouvoir, de la tradition, de la culture et de la légitimité : son seul nom suffit à évoquer, au-delà de la réalité géographique, un mode de vie citadin où les paradigmes religieux, identitaire et politique s'articulent pour produire une sorte d'espace fermement « strié ${ }^{20}$ ». On peut supposer que les écrivains étrangers ont largement contribué à l'élaboration de ce

20. À propos de cette notion d' « espace strié », opposée à celle d' « espace lisse », voir Gilles Deleuze et Félix Guattari, Mille Plateaux. Capitalisme et schizophrénie, t. II, Paris, Minuit, 1980, p. 592 et suiv. 
récit d'une ville figée dans le temps et portant les marques expressives d'un ordre. Cependant, ces paradigmes pénètrent jusque dans l'imaginaire des couches populaires marocaines pour lesquelles ils acquièrent une puissance symbolique. Dans une étude effectuée à partir d'un « corpus de 55 photos assemblées et collées successivement par trois générations de babouchiers sur un des murs à l'intérieur de leur espace de travail entre 1925 et 1987 à peu près ${ }^{21} "$, le politologue marocain Mohamed Tozy souligne l'importance de ces trois paradigmes y compris chez les corporations d'artisans. Rares sont aussi les textes écrits par des auteurs marocains qui ne fassent, d'une façon ou d'une autre, allusion à ce récit d'une ville incarnant une tradition millénaire de vie urbaine régie par ses propres lois et ses frontières. Cependant, comme tout Grand Récit, ce récit de Fès suscite des résistances, des contre-narrations, des dissensions et des dissidences. Bon nombre d'auteurs n'en investissent les emblèmes que pour mieux les critiquer et les faire voler en éclats. Accordant une attention particulière à l'individu et à ses représentations, ces auteurs mettent en crise les grands systèmes interprétatifs qui permettent de créer du symbolique à partir de lieux énonciatifs holistiques. Pour le narrateur de Driss Chraïbi, dans Le Passé simple, par exemple, Fès est avant tout une ville sacrée associée à la figure écrasante du père/Seigneur :

Je n'aime pas cette ville. Elle est mon passé et je n'aime pas mon passé. J'ai grandi, me suis émondé. Fès s'est ratatinée, tout simplement. [...]

Une maison, n'importe quelle boutique est un rejet brutal vers la matière. Ce n'est pas parce que Fès est vétuste ou que les éléments du siècle mécanique y sont à peine perceptibles; mais parce que cette ville dégage, si je puis dire, une odeur de sainteté qui imprègne les bâtiments, la mentalité des gens et l'atmosphère - une sainteté qui n'a pas de parenté avec celle des monastères ou des lieux de pèlerinages, mais faite de respect, de passivité que l'on pourrait avoir pour un ermite vieux de quelque mille ans. [...] Le Seigneur n'y est-il pas né 22 ?

Parmi tous les signifiants formant la ville en tant que texte urbain, ce sont la mosquée Qarawiyine et le mausolée Moulay Driss qui charrient le plus cette image d'une ville rigide, prise dans une doxa qui la décrit. Dans Le Passé simple, aucun des nombreux appels à la prière émanant des quatre coins de la ville ne parvient à noyer les appels imperturbables de ces deux lieux théologiques s'élevant au milieu d'une multitude d'autres bruits. Chez Fatéma Mernissi, c'est plutôt sur le mode de la transgression symbolique que la mosquée Qarawiyine est investie dans Rêves de femmes. Lorsque la narratrice monte, en compagnie de ses cousins Samir et Malika, sur la

21. Voir Mohamed Tozy, «L'imaginaire politique d'un babouchier de Fès. Essai de lecture sur un mur d'images ", dans A. Diouri (dir.), Les Puissances du symbole, Casablanca, Le Fennec, 1997, p. 77-90.

22. Driss Chaïbi, Le Passé simple, Paris, Denoël, 1954, p. 74-75. 
terrasse interdite, seul site du récit où on peut dominer la ville dans une vue panoramique, pour discuter du sens du mot harem, nous lisons :

Les minarets et même l'immense mosquée Quaraouiyine étaient à mes pieds, comme de minuscules jouets dans une ville en miniature. En même temps, les nuages au-dessus de ma tête me semblaient dangereusement proches, avec des flammes roses, presque rouges que je n'avais jamais distinguées d'en bas ${ }^{23}$.

Expérience singulière où l'allusion au mythe d'Icare parfaitement explicite avec l'usage de l'adverbe « dangereusement » et la référence à ces «flammes roses, presque rouges ». Néanmoins, c'est la manière dont Fatéma Mernissi investit les emblèmes de l'autorité religieuse, au nom de laquelle l'homme refuse l'accès à la cité à la femme, qui attire ici l'attention : se détachant du reste de la ville, les minarets et « l'immense mosquée Quaraouiyine » sont désormais aux pieds de la petite fille de sept ans comme de « minuscules jouets ». Si le changement d'échelle est à l'origine de cette miniaturisation des édifices religieux, il n'en épuise pas pour autant toute la portée symbolique. En effet, la contemplation ne prend son sens qu'à travers l'inversion des valeurs qu'elle engendre. Le regard reçoit des impressions de domination et s'exerce comme une action de «"gullivérisation" [où] ce qui devient petit nous rend grands ${ }^{24} \gg$.

Tahar Ben Jelloun, dans Harrouda, dénonce le rôle de l'université théologique, la Qarawiyine, dans la perpétuation des inégalités au sein d'une ville structurée par des discours généalogiques et identitaires ancrés dans la mémoire collective de ses habitants :

La société fassie se structura en fonction des inégalités décidées par une mémoire sacrée. L'artisan y a souffert. Méprisé. Rabaissé. Rejeté. Travailleur manuel, il n'avait pas de place dans la société qui se faisait. [...] Ville sans usines, Fassi de par sa vocation (!) de capitale intellectuelle a institué la différence entre la main et la pensée.

Le réseau des grandes familles s'est maintenu grâce au savoir dispensé à la Karaouiyne ou bien dans les écoles des fils de notables. Les fils d'artisans étaient destinés à apprendre et hériter le métier du père ${ }^{25}$.

Il est important de noter que ce discours identitaire, fonctionnant comme un support de vision/division qui construit l'espace urbain et l'organise selon des codes précis, circule dans les récits de plusieurs autres auteurs marocains. Des textes fondateurs des années 1970 à un roman comme Des houris et des hommes d'El Mostafa Bouignane ${ }^{26}$, un écrivain de la

23. Fatéma Mernissi, op.cit., p. 184.

24. Gaston Bachelard, La Terre et les Rêveries de la volonté [1947], Tunis, Cérès, 1996, p. $399-400$.

25. Tahar Ben Jelloun, Harrouda, Paris, Denoël, 1973, p. 85-86.

26. El Mostafa Bouignane, Des houris et des hommes, Rabat, Marsam, 2010. 
nouvelle génération, en passant par Rêves de femmes de Mernissi ou Sur ma mère ${ }^{27}$ de Ben Jelloun, il existe souvent dans les récits un « étranger » qui vient servir, selon l'un ou l'autre des personnages, de repoussoir au mode de vie qu'incarnent les « vrais » habitants de Fès. D'ailleurs, l'une des dichotomies qui circulent dans l'espace urbain de Fès, en y inscrivant des frontières tant matérielles qu'imaginaires, a trait à la distinction 'roubi (rural)/fassi. De fait, contrairement à ce qu'on pourrait croire,

dans la rhétorique de certains Fassi-s, la dénomination de 'roubi ne se limite pas [...] aux seuls migrants ruraux, elle caractérise des conduites et des façons de faire et de dire. Le 'roubi aussi serait toute personne sale, dépourvue de goût, de culture, de conscience civique et indifférente à la modernité. Le raffinement perpétuel des savoirs et savoir-faire dans l'art culinaire ou vestimentaire représente, en effet, pour certains Fassis d'origine une nécessité pour la pérennisation de la singularité de « leur» culture, et par conséquent, de «leur » identité ${ }^{28}$.

Dans Le Fond de la jarre d'Abdellatif Laâbi, par exemple, bien qu'il ne soit pas surdéterminé, ce modèle de la citadinité fassie fondé sur des artefacts langagiers et culturels est investi par des discours qui, en circulant dans l'espace urbain, organisent la ville selon une dynamique identitaire où le campagnard fonctionne comme l'un des plus grands marqueurs d'altérité. « Fès n'est plus ce qu'il était. Les campagnards l'ont envahi ${ }^{29}$ », se plaint d'ailleurs l'un des personnages du récit. De fait, c'est toute la représentation du narrateur et son appropriation de l'espace qui se trouvent affectées et contrôlées par ces postures identitaires, ce que montre cet extrait :

Les ateliers de la Source des Chevaux ont retrouvé leur animation. Celui des Ahl Touat, des maroquiniers originaires du Sahara, vibre de chants rythmés par des battements de mains. Namouss n'a jamais osé s'y aventurer. Les gens du Touat sont considérés un peu comme des étrangers, et donc tenus à l'écart. Mais leurs chants, si différents de ceux de Fès, sont d'une étrange beauté ${ }^{30}$.

\section{Les mutations de la ville entre nostalgie et réalisme}

Parallèlement à cette image d'une ville ancrée dans la tradition, Fès est aussi souvent représentée comme une ville en pleine mutation ou en dérive dont les valeurs et la culture sont sans cesse menacées et doivent donc être préservées. C'est dans ce sens d'ailleurs que s'inscrit l'entreprise de Laâbi

27. Tahar Ben Jelloun, Sur ma mère, Paris, Gallimard, 2008.

28. M'hammed Idrissi Janati, «Les images identitaires à Fès : divisions de la société, divisions de la ville », dans C. Topalov (dir.), Les Divisions de la ville, Paris, Éditions de la Maison des sciences de l'homme, 2002, p. 364.

29. Abdellatif Laâbi, op.cit., p. 28.

30. Ibid., p. 138. 
dans Le Fond de la jarre. Au-delà du simple réalisme autobiographique, la représentation de la ville dans ce récit consiste en " une exploration personnelle d'un héritage collectif livrant une perception de soi qui se situe dans les mouvements de la vie en société et le flux de l'histoire ${ }^{31} »$. De ce fait, les critères qui fondent la sélection des lieux, en vue de construire une cartographie littéraire de Fès, sont doubles : la maison familiale, la rue, le souk Sekkatine, Sidi Harazem, etc., ont une grande importance parce qu'ils sont liés à des expériences intimes qui ont marqué le narrateur, mais ils sont aussi des lieux où gît une culture à sauvegarder de l'oubli. Ben Jelloun, quant à lui, dans Harrouda, est beaucoup plus attentif à la modernisation de la ville, envahie par des bulldozers, dont il décrit la métamorphose, sous le protectorat, avec toute la lucidité de celui auquel l'histoire « impos[e] une nouvelle naissance ${ }^{32} \gg$. Aussi parle-t-il, sans nostalgie aucune, des « ruines qui sentaient encore l'odeur de l'intimité violée ${ }^{33}$ » et de « médina [qui] s'évanouissait au grand désespoir des touristes ${ }^{34} »$ friands d'exotisme. Cependant, c'est sous la plume de Paul Bowles, qui a vécu à Tanger de 1947 à sa mort en 1997, que Fès apparaît davantage comme une ville dont la culture traditionnelle est menacée. Pour ce romancier, Fès représente la ville du mystère et de l'étrange par excellence. Son premier contact avec la cité impériale est ainsi décrit dans son autobiographie :

We arrived in Fez at sunset and took a carriage through the Mellah to Fez-Djedid. Tangier had by no means prepared me for the experience of Fez, where everything was ten times stranger and bigger and brighter. I felt that I had left the world behind, and the resulting excitement was well-nigh unbearable ${ }^{35}$.

Cette attitude somme toute orientaliste de Paul Bowles vis-à-vis de Fès se manifeste de façon beaucoup plus explicite dans son roman La Maison de l'araignée $e^{36}$. Entamée au cours de l'été 1954, date significative dans la lutte du Maroc pour l'indépendance, cette fiction romanesque, qui se voulait au départ un hymne à la vie traditionnelle à Fès, est rattrapée par des événements politiques qui en modifient le cours comme l'explique l'auteur lui-même dans la postface. Le roman de Bowles nous livre une représentation de Fès complètement différente de celle qui prévaut dans les récits autobiographiques des auteurs marocains portant sur la même époque. Poursuivant simultanément le parcours de deux personnages, John

31. Safoi Babana-Hampton, Réflexions littéraires sur l'espace public marocain dans l'œuvre d'Abdellatif Laâbi, Birmingham, Summa Publications, 2008, p. 137.

32. Tahar Ben Jelloun, op. cit., p. 61.

33. Ibid., p. 62.

34. Ibid.

35. Paul Bowles, Without Stopping: An Autobiography [1972], New York, The Eco Press, 1985, p. 130.

36. Paul Bowles, La Maison de l'araignée [The Spider's House, 1955], Paris, Quai Voltaire, 1993. 
Stenham, un écrivain américain résidant au Palais Mérinides, et Amar, un jeune Fassi, l'auteur esquisse une cartographie de la ville vue à travers deux regards différents. Â la focalisation allogène ${ }^{37}$ de Stenham, doublant celle de Bowles, se superpose le point de vue endogène d'Amar, relayant autrement celui de l'auteur. Ainsi surgit une vision beaucoup plus large et modulée de la ville où les lieux valorisés se distinguent d'abord, selon les désirs, les contraintes et la mobilité de chacun, avant de finir par se confondre, sous la poussée du destin, dans un mouvement centripète de la narration faisant s'entrecroiser les parcours des deux personnages. Par bien des aspects littéraires, la représentation de Fès faite par Bowles, dans $L a$ Maison de l'araignée, est intéressante, mais elle demeure, pour une grande part, prisonnière des clichés orientalistes présents chez beaucoup d'écrivains voyageurs pour lesquels le voyage au Maroc constitue avant tout une façon de remonter le temps. Tout au long du roman, Stenham, en lequel le lecteur reconnaît immédiatement un porte-parole de l'auteur, ne cesse de relayer, dans une sorte de nostalgie romantique, un discours de regret et d'amertume face aux mutations qui menacent la ville et risquent de l'extirper de son état de cité médiévale, à l'abri du temps et de la modernisation. Pour ce personnage, peu importe que des gens vivant à Fès se plaignent « de l'humidité, de la saleté et de l'insalubrité38 ${ }^{38}$ et aient pour ambition de " créer un lieu mieux adapté à leur conception des nécessités modernes ", en cherchant à « ouvrir de larges avenues à travers les oliveraies ${ }^{39}$ » ou à « construire de hauts immeubles d'habitation ${ }^{40}$ »; la cité impériale est avant tout une œuvre d'art destinée au plaisir de tous les visiteurs étrangers. Mais Stenham sent que le désastre est imminent et que, compte tenu de la fascination des nationalistes pour tout ce qui est moderne et occidental, il est peutêtre en train de vivre les derniers moments d'une civilisation en agonie :

La ville cesserait pour toujours d'être ce qu'elle était. Quelques bombes suffiraient à transformer ses murs aux stucs délicats en amas ruinés de poussière blanche; ce ne serait plus le labyrinthe enchanté à l'abri de l'œuvre du temps où il pouvait errer sans pensée, laissant ses yeux lui dire qu'il avait enfin trouvé le chemin du retour. La chute de cette ville abolirait d'un coup le passé. Une brèche de mille ans se verrait comblée en une seconde par l'explosion de la première bombe. À partir de cet instant, jusqu'au moment

37. Le point de vue allogène est un point de vue intermédiaire entre l'exogène et l'endogène : " Il est le propre de tous ceux et toutes celles qui se sont fixés dans un endroit sans que celui-ci leur soit encore familier, sans non plus qu'il demeure pour eux exotique. Tel est le cas de Paul Bowles à Tanger, New-Yorkais d'origine, mais établi au partage de l'Atlantique et de la Méditerranée tout au long du demi-siècle qui court de 1947 à l'année de sa mort, en 1997. » (Bertrand Westphal, La Géocritique : réel, fiction, espace, Paris, Minuit, 2007, p. 209).

38. Paul Bowles, La Maison de l'araignée, op. cit., p. 198.

39. Ibid., p. 198.

40. Ibid., p. 191. 
où la métropole transformée s'étalerait, éclatante, entre ses boulevards et ses garages, tout se serait enchaîné comme un mécanisme inéluctable ${ }^{41}$.

On pourrait croire que la multifocalisation maintient un certain équilibre au sein de la fiction et que le point de vue endogène d'Amar sert de repoussoir à celui de Stenham. Rien de tel. Si Stenham, aveuglé par son désir orientaliste, en vient à faire une apologie du colonialisme créditant les Français d'avoir au moins " réussi à conserver Fès intact » en déclarant «l'ensemble de la ville "monument historique" 42 ", ce qui aurait rendu, du moins provisoirement, les « aspirations » des nationalistes " irréalisables ${ }^{43}$ », Amar apparaît comme un personnage fantasmatique cristallisant tout le désir orientaliste que l'Américain projette sur la ville. En effet, « si peu atteint par la présence d'une autre civilisation rationnelle, stérilisante ${ }^{44} »$, Amar n'est finalement là que pour apporter à Stenham « une consolation, une preuve vivante qu'on pouvait encore espérer remonter le cours du temps et assouvir le désir infantile de remonter aux origines ${ }^{45} \|$. Malgré cette vision somme toute orientaliste, Bowles décrit parfois, dans un réalisme cru, quelques-uns des maux qui rongent réellement Fès et menacent son paysage urbain, en nous rappelant que les mutations peuvent aussi être dégradations. La description d'un des nombreux misérables quartiers qui entouraient, déjà à l'époque, les murs de la cité, sous la poussée de l'exode, est significative à cet égard. Devant le spectacle du bidonville sordide, John Stenham évoque un nouveau désastre et note qu' « il y a quelques années, rien de tout ceci n'existait ${ }^{46} »$. Vingt-sept ans après la parution de La Maison de l'araignée, les propos de Bowles, revenant sur son expérience littéraire, font toujours écho à ceux de son personnage :

La ville de Fès existe toujours. Elle a cessé d'être le centre intellectuel et culturel de l'Afrique du Nord. Ce n'est qu'une ville de plus confrontée aux problèmes insolubles du tiers-monde ${ }^{47}$.

Cette image d'un Fès dégradé est aussi présente chez le jeune écrivain marocain El Mostafa Bouignane dans Des houris et des hommes. Très attentif aux signifiés actuels de la ville, ce roman, décrivant la cité impériale telle qu'elle apparaît à l'auteur plus de quarante ans après l'indépendance, nous livre une représentation particulière de Fès qui opère un déplacement dans le récit de sa centralité et en bouleverse toute la narration. L'auteur ancre l'intrigue de son récit à « la Source des Pèlerins », «l'un des faubourgs les

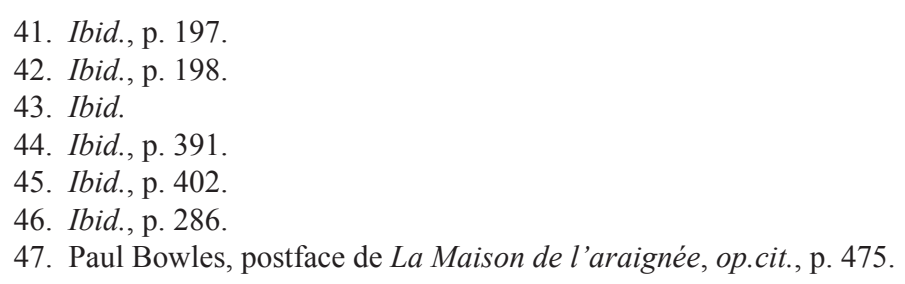


plus déshérités et les plus mal famés de la périphérie de Fès ${ }^{48}$ » où « des milliers de familles, chassées des campagnes environnantes par la sécheresse, $[\ldots]$ avaient échoué ${ }^{49} »$. Ainsi, contrairement à toute une littérature marocaine qui ne cesse de réduire, dans le cadre de l'autobiographie et des souvenirs d'enfance, la ville de Fès à sa médina, Des houris et des hommes construit plutôt la ville à partir d'une perception périphérique de l'espace urbain.

À l'instar de Mohamed Zafzaf, par exemple, le nouvelliste et romancier marocain arabophone dont les récits font toujours passer au premier plan Le Quartier-arrière ${ }^{50}$, le bidonville et le marginal, Bouignane opère ici un recadrage dans la représentation littéraire de la ville qui permet de dévoiler un nouveau type de paysage urbain souvent occulté. Malgré le métier de guide clandestin qu'exerce son narrateur, rares sont les moments où l'auteur décrit des lieux familiers et emblématiques de la ville. Son Fès est celui des cafés " grouill[ant] de jeunes désœuvrés ${ }^{51}$ ", des décharges publiques où des enfants passent « le plus clair de leur temps [...] à fouiller dans les montagnes d'immondices ${ }^{52} »$, des commissariats de police ou des centres pénitentiaires où règne la loi de la jungle. Voici comment le narrateur décrit, sur un ton ironique, sa ville, dont il découvre la réalité de plus près, le jour où il rend visite à sa petite amie Leïla dans un hôpital :

De temps à autre, des taxis et des ambulances déglinguées, sans autre équipement qu'un brancard, s'arrêtaient sur l'aire des urgences, dégorgeaient des patients. Des blessés, victimes, pour la plupart, d'agressions à l'arme blanche. Fès by night. Devant l'entrée du service, le sol était maculé de sang. Je m'assis sur un banc et pensai aux inepties que j'avais débitées pendant toute la journée à un groupe de touristes : Fès, capitale spirituelle du Royaume, blabl-bla-a. Cité de paix, de sagesse, de tolérance, blabl-bla-a... Et la Fès qui se cache derrière ce joli conte? Celle des bas-fonds sombres où règnent la misère sordide, le vol, la prostitution et les crimes crapuleux. La capitale spirituelle aux milliers d'enfants analphabètes, parce que non scolarisés, qui se livrent dès l'âge le plus tendre à la mendicité ou à la rapine. La cité de la sagesse aux milliers d'adolescents livrés à eux-mêmes, qui, sous l'empire du qarqoubi - puissant psychotrope bon marché qui dévaste le cerveau - se tailladent le torse et les avant-bras à coups de lames de rasoir? La ville de la paix aux innombrables gangs armés qui sévissent dans les bas quartiers ${ }^{53} \ldots$

48. El Mostafa Bouignane, op. cit., p. 8.

49. Ibid.

50. Titre d'un roman de Mohamed Zafzaf (1992) qui a par ailleurs publié d'autres textes comme La Vipère et la Mer (1979), L'Euf du coq (1984), ou Tentative de vie (1985).

51. El Mostafa Bouignane, op. cit., p. 9.

52. Ibid., p. 28.

53. Ibid., p. 149. 
Cet effritement du Grand Récit de la ville, que l'auteur ne cesse de souligner dès l'incipit de son roman, n'est pas vécu de la même façon par tous les personnages. Pour un « vieux fassi facho », par exemple, avec qui le narrateur se retrouve enfermé, le temps d'un trajet, dans un taxi et qu'il écoute « déblatérer » en silence, l'état actuel de la ville est à la fois l'occasion d'évoquer, sur un ton nostalgique, le Fès d'antan et de s'indigner contre toute « la racaille qui a envahi la ville ${ }^{54} »$ la rendant inhabitable. Cependant, pour Bouignane, le choix du Fès des «bas-fonds » comme lieu d'énonciation littéraire est surtout un acte politiquement surdéterminé qui vise à reconstruire la ville en contrepoint de tous les discours officiels et imaginaires pétris de clichés et d'évidences. De fait, depuis le début jusqu'à la fin, la représentation littéraire de Fès dans le roman, qui s'ouvre, par ailleurs, sur la retransmission en direct des funérailles de Hassan II, se déploie comme un repoussoir au commentaire du speaker, «glorifiant le Royaume prospère et bienheureux » que le monarque « léguait à son successeur». Selon une scénographie qui fonde sa propre légitimation sur une rupture, effectuée sur le mode de l'ironie, avec cette vision paradisiaque du pays, la suite du roman ne cessera de tourner en dérision les propos du speaker. En outre, l'auteur opère une projection de l'espace urbain comme un théâtre où la misère et l'insécurité se révèlent être ce qui nourrit un discours islamiste radical; ce qui lui permet de déconstruire un aspect fondamental du récit de la centralité de la ville lié à son image de capitale spirituelle du royaume. Le Fès religieux du roman n'est ni celui de la mosquée Qarawiyine, ni celui des ulémas officiels; il est plutôt associé à un discours mystificateur, produit à partir de lieux périphériques et clandestins, qui modifie à la fois le comportement du sujet urbain et le paysage de la ville prise dans une nouvelle configuration sensorielle codée et décodée à partir du paradigme du sacré :

Un café où l'on fumait le haschisch fut brûlé un soir. [...] Beaucoup de jeunes se mirent à fréquenter la mosquée et se laissèrent pousser la barbe. Bien des filles prirent le voile [...].

Sur les trottoirs et dans de nombreuses échoppes du quartier fleurit un commerce d'articles religieux : exemplaires du Coran de différentes tailles, petits livres d'invocations ou d'initiation à la prière, cassettes de prédicateurs célèbres [...]. Dans l'air, fusant de magnétophones mis à plein volume, se confondaient des voix qui psalmodiaient des versets coraniques avec celles des prédicateurs [...].

Chaque soir, une foule bariolée se promenait entre éventaires et étalages. Des femmes voilées de la tête aux pieds. Gantées. Des hommes vêtus d'amples tuniques afghanes, les yeux noircis au khôl, la barbe cascadant jusqu'au nombril et la tête auréolée de gros turbans. Au spectacle de ces mollahs déambulant dans nos rues comme en territoire conquis, parmi les ânes, les carrioles et les tentes crasseuses, avec le mont Zalagh et un ciel plombé en toile de fond, on se serait cru à Kandahar ${ }^{55}$.

54. Ibid., p. 79.

55. Ibid., p. 10-11. 
Ce qui montre peut-être le plus cette action du discours islamiste sur l'espace publique en tant que texte urbain dont il transforme violemment les signes, ce sont les graffitis découverts un jour sur le mur du café California qualifiant le lieu de « repaire de moulhidine et de kouffar - athées et mécréants ${ }^{56} \gg$ (p. 104); ce qui pousse d'ailleurs le patron à abandonner le nom américain et à choisir un nouveau nom imprégné d'une aura de sacré pour son estaminet : Café Bismillah.

La représentation littéraire de la ville oscille entre le global et le singulier, à la manière des « rhétoriques piétonnières ${ }^{57}$ » qu'évoque Michel de Certeau lorsqu'il s'intéresse aux pratiques singulières de l'espace et aux façons dont les individus réinventent les systèmes en les déjouant. En effet, selon l'anthropologue Marc Augé, en parlant de « rhétoriques piétonnières » pour qualifier la production de l'espace du point de vue des «pratiques microbiennes, singulières et plurielles ${ }^{58} »$, de Certeau suggère que « les contraintes syntaxiques [...] ne sont pas contradictoires avec la liberté de l'écriture, avec le style défini comme maniement personnel et singulier de la langue, que l'imaginaire individuel peut se frayer un chemin entre les symboles urbains ${ }^{59}$ ».

En somme, la représentation littéraire de Fès n'évoque pas l'image d'une ville inerte et figée dans le temps, mais plutôt le récit d'une cité décomposée et recomposée au gré des genres, des sexes et des époques. Comme autant de regards singuliers posés sur la ville, les œuvres littéraires, tantôt se rencontrant tantôt divergeant, ne cessent de réinventer, chacune à sa manière, le paysage de la cité impériale et son Grand Récit en en opérant à chaque fois un nouvel agencement littéraire nous livrant, à l'interface des connexions entre réalité et littérature, une image toujours mouvante et décentrée de la ville. Le Fès littéraire est à la fois un et pluriel : c'est le produit de pratiques urbaines et de représentations collectives réduisant la ville à ses repères presque incontournables et à ses symboles; mais c'est aussi une ville singulière que chaque écrivain décrit et restitue à la manière d'un flâneur solitaire choisissant son propre itinéraire.

\section{Anouar Ouyachchi \\ Université Moulay Ismaïl - Meknès \\ Équipe d'études culturelles et postcoloniales}

56. Ibid., p. 104.

57. Voir Marc Augé, Pour une anthropologie des mondes contemporains, Paris, Flammarion, 1997, p. 133 et suiv.

58. Michel de Certeau cité par Marc Augé, op. cit., p. 133.

59. Ibid., p. 160. 